\title{
On a class of boundary value problems under $A B C$ fractional derivative
}

\author{
Rozi Gul', Kamal Shah', Zareen A. Khan ${ }^{2 *}$ and Fahd Jarad ${ }^{3,4^{*}}$ (B)
}

\begin{tabular}{l}
\hline${ }^{*}$ Correspondence: \\
dr.zareenkhan@ymail.com; \\
fahd@cankaya.edu.tr \\
2Department of Mathematical \\
Sciences, Faculty of Science, \\
Princess Nourah bint Abdulrahman \\
University, P.O. Box 84428, Riyadh \\
11671, Saudi Arabia \\
${ }^{3}$ Department of Mathematics, \\
Cankaya University, Ankara, Turkey \\
Full list of author information is \\
available at the end of the article
\end{tabular}

\begin{abstract}
In this work, we establish some necessary results about existence theory to a class of boundary value problems (BVPs) of hybrid fractional differential equations (HFDEs) in the frame of Atangana-Baleanu-Caputo $(A B C)$ fractional derivative. Making use of Krasnoselskii and Banach theorems, we obtain the required conditions. Some appropriate results of Hyers-Ulam $(\mathrm{H}-\mathrm{U})$ stability corresponding to the considered problem are also established. Also a pertinent example is given to demonstrate the results.
\end{abstract}

MSC: 34A08; 47H08; 34A38; 05B07; 34K20

Keywords: $A B C$ fractional order derivative; HFDEs; Krasnoselskii and Banach theorems; Stability

\section{Introduction}

In previous decades, the area of fractional calculus got much attention from researchers. This is because of numerous applications of fractional order differential equations (FDEs) in mathematical modeling of real world phenomena (see [1-5]). The researchers therefore considered FODEs in their research a lot in the last many years. They established various analyses for the mentioned area including qualitative results for the existence of solution, numerical and stability results, etc. For the respective analysis of FODEs, the researchers used different tools like fixed point approach, Picard type theory, etc. In this respect, huge research work has been established, for details we refer to [6-9].

Here we remark that a class formed from quadratic perturbations of nonlinear differential equations called hybrid differential equations has got proper attention. In this regard, Dhage and Lakshmikantham [10] established significant results and theories. The respective existence theory of solution to the mentioned area is very important and can be established by using hybrid type fixed point approach. As it is known, fractional calculus is more realistic to describe many real world problems including memory and hereditary properties of various materials and processes as compared to classical calculus. Therefore researchers have extended the idea of fractional calculus to investigate H-FDEs from different aspects including existence results and stability theory. In this regard, various interesting results have been established in the literature (see [11-18]). Nowadays, fractional partial differential equations have received a great deal of attention in both analysis and

(c) The Author(s) 2021. This article is licensed under a Creative Commons Attribution 4.0 International License, which permits use, sharing, adaptation, distribution and reproduction in any medium or format, as long as you give appropriate credit to the original author(s) and the source, provide a link to the Creative Commons licence, and indicate if changes were made. The images or other third party material in this article are included in the article's Creative Commons licence, unless indicated otherwise in a credit line to the material. If material is not included in the article's Creative Commons licence and your intended use is not permitted by statutory regulation or exceeds the permitted use, you will need to obtain permission directly from the copyright holder. To view a copy of this licence, visit http://creativecommons.org/licenses/by/4.0/. 
application, and they are used to model various phenomena in diverse areas of science, for instance, chemistry, biology, physics, control theory, and engineering (see [17, 19-21]).

Fractional order derivative has been defined in various ways. Since the mentioned derivative is in fact a definite integral, including its integer counterpart is a special case. Therefore various definitions have been given in the literature in the last decades. Among these definitions, the Riemann-Liouville and Caputo ones have been used in plenty of research articles. Since these definitions involve a singular type kernel, it often makes some complication in numerical treatment of some problems. Therefore very recently Caputo and Fabrizio have defined a new differential operator by replacing singular kernel with nonsingular (exponential type) kernel (we refer to [22]). In subsequent years Atangana and Baleanu introduced a more general definition than Caputo-Fabrizio by including MittagLeffler (ML) type kernel (abbreviated as $A B C$ derivative of fractional order). The concerned derivative got much attention and has been used in plenty of research works (see [23-26]). Using the $A B C$ derivative of fractional order, various problems have been studied for existence and stability results, we refer to [27, 28]. In addition, generalized ML kernel operators have been studied by many researchers in applied fields of engineering and sciences. Such kernel types of operators are nonsingular ML kernels with one parameter and also arbitrary ML kernel parameters studied in [29]. Furthermore, some fractional operators have been studied with ML kernels (see [30]). On the other hand, intuitively, fixed point theory can be used in various fields of science and engineering as an authoritative modeling tool to obtain experimental solutions and results. From a broader point of view, the fixed point theory can also be seen as an attempt to link the biological or computational science research on innumerable abstract spaces, on convergence analysis, and on compactness [31]. Further, fixed point theorems are the main core for obtaining the necessary and sufficient conditions for the existence and uniqueness of fractional differential equations. In particular, Banach's fixed point theorem is essential for the uniqueness of the solution, but it needs a strong hypothesis to be applied (see [32, 33]). Some authors may use a priori limits in system solutions to prove the existence of solutions (see [34, 35]).

Motivated by the aforementioned work, in this article we investigate the following BVP of H-FDEs under the $A B C$ derivative of fractional order:

$$
\left\{\begin{array}{l}
{ }_{0}^{A B C} \mathrm{D}_{t}^{\omega}[\phi(t)-\mathscr{F}(t, \phi(t))]=\mathscr{G}(t, \phi(t)), \quad 0<\omega \leq 1, t \in[0, \xi]=\mathscr{I}, \\
\phi(0)=\int_{0}^{\xi} \frac{(\xi-\eta)^{\omega-1}}{\Gamma(\omega)} \mathscr{H}(\eta, \phi(\eta)) d \eta
\end{array}\right.
$$

where

$$
\mathscr{F}, \mathscr{G}, \mathscr{H}: \mathscr{I} \times \mathrm{R} \rightarrow \mathrm{R}
$$

Here we state that the considered problem is subjected to the nonlinear integral boundary condition (IBC). The BVPs have many applications which can be traced out in the applied mathematical sciences. For instance, in the theory of thermal ignition of gases, nonlinear diffusion generated by nonlinear sources, and concentration in chemical or biological problems. Further BVPs under IBCs have various applications of mathematical modeling in thermal conduction process, hemic conduction procedure, and hydrodynamics problems, see [36, 37]. Keeping in mind the importance of integral BVPs, in this paper, we study 
the aforesaid considered problem (1) for qualitative analysis of existence and stability results of Ulam type. The concerned $\mathrm{H}-\mathrm{U}$ stability has been investigated for usual FODEs in detail, see [38-40]. So far the mentioned stability has been very rarely considered for $\mathrm{H}$-FODEs under $A B C$ derivative of fractional order. We use fixed point approach due to Krasnoselskii [41] and Banach's contraction principle to derive sufficient results for the existence of at least one solution and unique solution. Further, using the concepts of nonlinear analysis, we also establish stability results of $\mathrm{H}-\mathrm{U}$ type. Finally, a pertinent example is given to justify the results.

This paper is organized as follows: The first part contains a detailed introduction. The second part contains basic results. The third portion is devoted to existence results. Portion four is related to stability results. In the fifth portion, we give an example, and the last part is related to brief conclusion.

\section{Axillary results}

In this section, we study some fundamental results, which are used in our main work.

Definition 1 ([42]) Let $w \in H^{1}(0, \xi)$ with $\omega \in(0,1]$, the $A B C$ derivative of fractional order is defined as

$$
{ }_{0}^{A B C} \mathrm{D}_{t}^{\omega} w(t)=\frac{\mathcal{N}(\omega)}{1-\omega} \int_{0}^{t} \frac{d w}{d \eta} \mathscr{E}_{\omega}\left[\frac{-\omega(t-\eta)}{1-\omega}\right] d \eta,
$$

where $\mathscr{E}_{\omega}=\sum_{i=0}^{\infty} \frac{t^{i \omega}}{\Gamma(\omega i+1)}$ is a Mittag-Leffler function. Also $\mathcal{N}(\omega)$ is called normalization function.

Definition 2 ([43]) The corresponding integral is expressed for $w$ as

$$
{ }_{0}^{A B} \mathrm{I}_{t}^{\omega} w(t)=\frac{(1-\omega)}{\mathcal{N}(\omega)} w(t)+\frac{\omega}{\mathcal{N}(\omega)} \int_{0}^{t} \frac{(t-\eta)^{\omega-1}}{\Gamma(\omega)} w(\eta) d \eta
$$

Lemma 1 ([44]) The solution of

$$
\begin{aligned}
& { }_{0}^{A B C} \mathrm{D}_{t}^{\omega} \phi(t)=\kappa(t), \\
& \phi(0)=\phi_{0},
\end{aligned}
$$

with the conditions such that the right-hand side vanishes at $t=0$ is given by

$$
\phi(t)=\phi_{0}+\frac{(1-\omega)}{\mathcal{N}(\omega)} \kappa(t)+\frac{\omega}{\mathcal{N}(\omega) \Gamma(\omega)} \int_{0}^{t}(t-\eta)^{\omega-1} \kappa(\eta) d \omega .
$$

Here we denote by $\mathscr{Z}=A C(\mathscr{J})$ the Banach space under the norm $\|\phi\|_{Z}=\max _{t \in \mathscr{J}}|\phi(t)|$.

Theorem 1 ([45]) Let for a convex set $\mathscr{B}$ of $\mathscr{Z}$ with a mapping $\mathscr{A} w=F w+G u$ such that

1. $F w+G u$ for every $u, w \in \mathscr{B}$;

2. $F$ is compact and continuous;

3. $G$ is contraction,

then $\mathscr{A}$ has at least one fixed point. 


\section{Main results}

In this part, we investigate conditions for the existence of at least one solution to H-FDEs (1).

Lemma 2 If $\kappa \in L(\mathscr{J})$, then the solution $\phi \in A C(\mathscr{J})$ of linear BVPs with nonlinear integral BCs

$$
\left\{\begin{array}{l}
{ }_{0}^{A B C} \mathrm{D}_{t}^{\omega} \phi(t)=\kappa(t), \quad 0<\omega \leq 1, t \in \mathscr{J}, \\
\phi(0)=\int_{0}^{\xi} \frac{(\xi-\eta)^{\omega-1}}{\Gamma(\omega)} \mathscr{H}(\eta, \phi(\eta)) d \eta
\end{array}\right.
$$

is given by

$$
\begin{aligned}
\phi(t)= & \int_{0}^{\xi} \frac{(\xi-\eta)^{\omega-1}}{\Gamma(\omega)} \mathscr{H}(\eta, \phi(\eta)) d \eta+\frac{(1-\omega)}{\mathcal{N}(\omega)} \kappa(t) \\
& +\frac{\omega}{\mathcal{N}(\omega) \Gamma(\omega)} \int_{0}^{t}(t-\eta)^{\omega-1} \kappa(\eta) d \eta .
\end{aligned}
$$

Proof 1 Thanks to Lemma 1, we can easily obtain result (2).

Corollary 1 In view of Lemma 2, the solution of nonlinear integral BVPs (1) is given by

$$
\begin{aligned}
\phi(t)= & \mathscr{F}(t, \phi(t))+\frac{(1-\omega)}{\mathcal{N}(\omega)} \mathscr{G}(t, \phi(t))+\int_{0}^{\xi} \frac{(\xi-\eta)^{\omega-1}}{\Gamma(\omega)} \mathscr{H}(\eta, \phi(\eta)) d \eta \\
& +\frac{\omega}{\mathcal{N}(\omega) \Gamma(\omega)} \int_{0}^{t}(t-\eta)^{\omega-1} \mathscr{G}(\eta, \phi(\eta)) d \eta .
\end{aligned}
$$

To derive the interrelated results, we need the following hypothesis to hold:

$\left(A_{1}\right)$ For constants $L_{\mathscr{F}}, L_{\mathscr{G}}, L_{\mathscr{H}}>0$, we have for any $\phi, \bar{\phi} \in \mathscr{Z}$

$$
\begin{aligned}
& |\mathscr{F}(t, \phi(t))-\mathscr{F}(t, \bar{\phi}(t))| \leq L_{\mathscr{F}}|\phi(t)-\bar{\phi}(t)|, \\
& |\mathscr{G}(t, \phi(t))-\mathscr{G}(t, \bar{\phi}(t))| \leq L_{\mathscr{G}}|\phi(t)-\bar{\phi}(t)|,
\end{aligned}
$$

and

$$
|\mathscr{H}(t, \phi(t))-\mathscr{H}(t, \bar{\phi}(t))| \leq L_{\mathscr{H}}|\phi(t)-\bar{\phi}(t)| .
$$

$\left(A_{2}\right)$ For any real constants $C_{\mathscr{G}}, M_{\mathscr{G}}>0$, we have

$$
|\mathscr{G}(t, \phi(t))| \leq C_{\mathscr{G}}|\phi(t)|+M_{\mathscr{G}} .
$$

$\left(A_{3}\right)$ Also, for real constants $C_{\mathscr{H}}>0, M_{\mathscr{H}}>0$, we have

$$
|\mathscr{H}(t, \phi(t))| \leq C_{\mathscr{H}}|\phi(t)|+M_{\mathscr{H}} .
$$


On using (3), we define the operator $\mathscr{A}: \mathscr{Z} \rightarrow \mathscr{Z}$ by

$$
\begin{aligned}
\mathscr{A} \phi(t)= & \mathscr{F}(t, \phi(t))+\frac{(1-\omega)}{\mathcal{N}(\omega)} \mathscr{G}(t, \phi(t))+\int_{0}^{\xi} \frac{(\xi-\eta)^{\omega-1}}{\Gamma(\omega)} \mathscr{H}(\eta, \phi(\eta)) d \eta \\
& +\frac{\omega}{\mathcal{N}(\omega) \Gamma(\omega)} \int_{0}^{t}(t-\eta)^{\omega-1} \mathscr{G}(\eta, \phi(\eta)) d \eta, \quad t \in \mathscr{J} .
\end{aligned}
$$

Theorem 2 Under hypothesis $\left(A_{1}\right)$, the considered problem (1) has a unique solution if

$$
\Upsilon=\left(L_{\mathscr{F}}+\frac{(1-\omega)}{\mathcal{N}(\omega)} L_{\mathscr{G}}+\frac{L_{\mathscr{H}} \xi^{\omega}}{\Gamma(\omega+1)}\left(1+\frac{\omega}{\mathcal{N}(\omega)}\right)\right)<1
$$

Proof 2 If $\phi, \bar{\phi} \in \mathscr{Z}$, from (4) we have

$$
\begin{aligned}
\|\mathscr{A}(\phi)-\mathscr{A}(\bar{\phi})\|_{\mathscr{Z}}= & \max _{t \in \mathscr{J}}|\mathscr{A} \phi(t)-\mathscr{A} \bar{\phi}(t)| \\
= & \max _{t \in \mathscr{J}} \mid \mathscr{F}(t, \phi(t))+\frac{(1-\omega)}{\mathcal{N}(\omega)} \mathscr{G}(t, \phi(t)) \\
& +\int_{0}^{\xi} \frac{(\xi-\eta)^{\omega-1}}{\Gamma(\omega)} \mathscr{H}(\eta, \phi(\eta)) d \eta \\
& +\frac{\omega}{\mathcal{N}(\omega) \Gamma(\omega)} \int_{0}^{t}(t-\eta)^{\omega-1} \mathscr{G}(\eta, \phi(\eta)) d \eta \\
& -\mathscr{F}(t, \bar{\phi}(t))-\frac{(1-\omega)}{\mathcal{N}(\omega)} \mathscr{G}(t, \bar{\phi}(t)) \\
& -\int_{0}^{\xi} \frac{(\xi-\eta)^{\omega-1}}{\Gamma(\omega)} \mathscr{H}(\eta, \bar{\phi}(\eta)) d \eta \\
& -\frac{\omega}{\mathcal{N}(\omega) \Gamma(\omega)} \int_{0}^{t}(t-\eta)^{\omega-1} \mathscr{G}(\eta, \bar{\phi}(\eta)) d \eta \mid \\
\leq & L \mathscr{F}\|\phi-\bar{\phi}\| \mathscr{Z}+\frac{(1-\omega)}{\mathcal{N}(\omega)} L \mathscr{G}\|\phi-\bar{\phi}\|_{\mathscr{Z}} \\
& +\frac{L \mathscr{H} \xi^{\omega}}{\Gamma(\omega+1)}\|\phi-\bar{\phi}\| \mathscr{Z}+\frac{\omega L \mathscr{H} \xi^{\omega}}{\mathcal{N}(\omega) \Gamma(\omega+1)}\|\phi-\bar{\phi}\|_{\mathscr{Z}} .
\end{aligned}
$$

Hence, we obtain

$$
\|\mathscr{A}(\phi)-\mathscr{A}(\bar{\phi})\|_{\mathscr{Z}} \leq\left(L_{\mathscr{F}}+\frac{(1-\omega)}{\mathcal{N}(\omega)} L_{\mathscr{G}}+\frac{L_{\mathscr{H}} \xi^{\omega}}{\Gamma(\omega+1)}\left(1+\frac{\omega}{\mathcal{N}(\omega)}\right)\right)\|\phi-\bar{\phi}\|_{\mathscr{Z}}
$$

Thus we get

$$
\|\mathscr{A}(\phi)-\mathscr{A}(\bar{\phi})\|_{\mathscr{Z}} \leq \Upsilon\|\phi-\bar{\phi}\|_{\mathscr{Z}}
$$

Therefore, $\mathscr{A}$ is a contraction and the said problem (1) has unique solutions.

Thanks to Theorem 1, we derive the next result. 
Theorem 3 Thanks to hypotheses $\left(A_{1}\right)$ and $\left(A_{2}\right)$, the H-FDE (1) has at least one solution if

$$
L_{\mathscr{F}}+\frac{(1-\omega)}{\mathcal{N}(\omega)} L_{\mathscr{G}}<1
$$

Proof 3 Here we define two operators $F$ and $G$ from (4) as

$$
\begin{aligned}
F \phi(t)= & \mathscr{F}(t, \phi(t))+\frac{(1-\omega)}{\mathcal{N}(\omega)} \mathscr{G}(t, \phi(t)), \\
G \phi(t)= & \int_{0}^{\xi} \frac{(\xi-\eta)^{\omega-1}}{\Gamma(\omega)} \mathscr{H}(\eta, \phi(\eta)) d \eta \\
& +\frac{\omega}{\mathcal{N}(\omega) \Gamma(\omega)} \int_{0}^{t}(t-\eta)^{\omega-1} \mathscr{G}(\eta, \phi(\eta)) d \eta .
\end{aligned}
$$

Step 1 . Let $\phi, \bar{\phi} \in \mathscr{Z}$, we have from (6)

$$
|F \phi(t)-F \bar{\phi}(t)| \leq|\mathscr{F}(t, \phi(t))-\mathscr{F}(t, \bar{\phi}(t))|+\left|\frac{(1-\omega)}{\mathcal{N}(\omega)}(\mathscr{G}(t, \phi(t))-\mathscr{G}(t, \bar{\phi}(t)))\right|
$$

On simplification, we obtain

$$
\|F \phi-F \bar{\phi}\|_{\mathscr{Z}} \leq L_{\mathscr{F}}\|\phi-\bar{\phi}\|_{\mathscr{Z}}+\frac{(1-\omega)}{\mathcal{N}(\omega)} L \mathscr{G}\|\phi-\bar{\phi}\|_{\mathscr{Z}}
$$

We have

$$
\|F \phi-F \bar{\phi}\|_{\mathscr{Z}} \leq\left(L_{\mathscr{F}}+\frac{(1-\omega)}{\mathcal{N}(\omega)} L \mathscr{G}\right)\|\phi-\bar{\phi}\|_{\mathscr{Z}}
$$

Thus $F$ is contraction.

Step 2 . To derive the required condition in respect of $G$, let $\mathscr{B}=\left\{\phi \in \mathscr{Z} ;\|\phi\|_{\mathscr{Z}} \leq \rho\right\}$, then from (6) we can do

$$
\begin{aligned}
& |G \phi(t)| \leq\left|\int_{0}^{\xi} \frac{(\xi-\eta)^{\omega-1}}{\Gamma(\omega)} \mathscr{H}(\eta, \phi(\eta)) d \eta\right|+\left|\frac{\omega}{\mathcal{N}(\omega) \Gamma(\omega)} \int_{0}^{t}(t-\eta)^{\omega-1} \mathscr{G}(\eta, \phi(\eta)) d \eta\right| \\
& \leq \int_{0}^{\xi} \frac{(\xi-\eta)^{\omega-1}}{\Gamma(\omega)}|\mathscr{H}(\eta, \phi(\eta))| d \eta+\frac{\omega}{\mathcal{N}(\omega) \Gamma(\omega)} \int_{0}^{t}(t-\eta)^{\omega-1}|\mathscr{G}(\eta, \phi(\eta))| d \eta, \\
& \|G \phi\|_{\mathscr{Z}} \leq \frac{\xi^{\omega}}{\Gamma(\omega+1)}\left(C_{\mathscr{H}}\|\phi\|_{\mathscr{Z}}+M_{\mathscr{H}}\right)+\frac{\omega \xi^{\omega}}{\mathcal{N}(\omega) \Gamma(\omega+1)}\left(C_{\mathscr{G}}\|\phi\|_{\mathscr{Z}}+M_{\mathscr{G}}\right), \\
& \|G \phi\|_{\mathscr{Z}} \leq \frac{\xi^{\omega}}{\Gamma(\omega+1)}\left(C_{\mathscr{H}} \rho+M_{\mathscr{H}}+\frac{\omega}{\mathcal{N}(\omega)}\left(C_{\mathscr{G} \rho}+M_{\mathscr{G}}\right)\right)=\Lambda \\
& \|G \phi\|_{\mathscr{Z}} \leq \Lambda \text {. }
\end{aligned}
$$

Thus $G$ is bounded. Further, as $\mathscr{H}, \mathscr{G}$ are continuous, therefore $G$ is also. 
Step 3. For equicontinuity, let $t_{1}<t_{2} \in \mathscr{J}$, we have

$$
\begin{aligned}
\left|G \phi\left(t_{2}\right)-G \phi\left(t_{1}\right)\right| \leq & \mid \frac{\omega}{\mathcal{N}(\omega) \Gamma(\omega)} \int_{0}^{t_{2}}\left(t_{2}-\eta\right)^{\omega-1} \mathscr{G}(\eta, \phi(\eta)) d \eta \\
& -\frac{\omega}{\mathcal{N}(\omega) \Gamma(\omega)} \int_{0}^{t_{1}}\left(t_{1}-\eta\right)^{\omega-1} \mathscr{G}(\eta, \phi(\eta)) d \eta \mid \\
\leq & \left|\frac{\omega}{\mathcal{N}(\omega) \Gamma(\omega)} \int_{0}^{t_{1}}\left[\left(t_{2}-\eta\right)^{\omega-1}-\left(t_{2}-\eta\right)^{\omega-1}\right] \mathscr{G}(\eta, \phi(\eta)) d \eta\right| \\
& +\left|\frac{\omega}{\mathcal{N}(\omega) \Gamma(\omega)} \int_{t_{1}}^{t_{2}}\left(t_{2}-\eta\right)^{\omega-1} \mathscr{G}(\eta, \phi(\eta)) d \eta\right| \\
\leq & \frac{\omega}{\mathcal{N}(\omega) \Gamma(\omega+1)}\left(C_{\mathscr{G} \rho} \rho M_{\mathscr{G}}\right)\left(t_{1}^{\omega}-t_{2}^{\omega}\right) .
\end{aligned}
$$

Obviously, from (7), we see that $t_{1} \rightarrow t_{2}$, the right-hand side of the above inequality goes to zero, therefore $\left\|G \phi\left(t_{2}\right)-G \phi\left(t_{1}\right)\right\|_{\mathscr{Z}} \rightarrow 0$ as $t_{1} \rightarrow t_{2}$. As the operator $G \phi$ is continuous and bounded, so it is uniformly continuous. Also $G(\mathscr{B}) \subset \mathscr{B}$ is compact. Thanks to ArzeláAscoli theorem, the operator $G$ fulfills all the conditions of complete continuity. Hence H-FDE (1) has at least one solution.

\section{Stability results}

This portion is devoted to establishing results about $\mathrm{H}-\mathrm{U}$ stability for considered $\mathrm{H}-\mathrm{FDE}$ (1).

Definition $3 \mathrm{H}-\mathrm{FDE}(1)$ is $\mathrm{H}-\mathrm{U}$ stable if, for any $\varepsilon>0$, for the given inequality

$$
\left|{ }_{0}^{A B C} \mathrm{D}_{t}^{\omega}[\phi(t)-\mathscr{F}(t, \phi(t))]-\mathscr{G}(t, \phi(t))\right|<\varepsilon \quad \text { for all } t \in \mathscr{J},
$$

there exists the unique solution $\bar{\phi}$ with a constant $\mathscr{W}_{\mathscr{F}}$ such that

$$
|\phi(t)-\bar{\phi}(t)| \leq \mathscr{W}_{\mathscr{F} \mathcal{E}} \quad \text { for all } t \in \mathscr{J} .
$$

Further, $\mathrm{H}$-FDE problem (1) will be generalized $\mathrm{H}-\mathrm{U}$ stable if there exists a nondecreasing function $\varphi:(0,1) \rightarrow(0, \infty)$ such that

$$
|\phi(t)-\bar{\phi}(t)| \leq \mathscr{W}_{\mathscr{F}} \varphi(\varepsilon) \quad \text { for all } t \in \mathscr{J},
$$

with $\varphi(0)=0$.

The given result is needed.

Remark 1 If we have a function $\psi$ independent of $\phi \in \mathscr{Z}$ with $\psi(0)=0$ and $\psi(\xi)=0$ with

1. $|\psi(t)| \leq \varepsilon$ for every $t \in \mathscr{J}$;

2. ${ }_{0}^{A B C} \mathrm{D}_{t}^{\omega}[\phi(t)-\mathscr{F}(t, \phi(t))]=\mathscr{G}(t, \phi(t))+\psi(t)$ for all $t \in \mathscr{J}$.

Lemma 3 The solution of the given H-FDE problem

$$
\left\{\begin{array}{l}
{ }_{0}^{A B C} \mathrm{D}_{t}^{\omega}[\phi(t)-\mathscr{F}(t, \phi(t))]=\mathscr{G}(t, \phi(t))+\psi(t), \quad 0<\omega \leq 1, t \in \mathscr{J}, \\
\phi(0)=\int_{0}^{\xi} \frac{(\xi-\eta)^{\omega-1}}{\Gamma(\omega)} \mathscr{H}(\eta, \phi(\eta)) d \eta
\end{array}\right.
$$


is

$$
\begin{aligned}
\phi(t)= & \left.\mathscr{F}(t, \phi(t))+\frac{(1-\omega)}{\mathcal{N}(\omega)} \mathscr{G}(t, \phi(t))+\frac{(1-\omega)}{\mathcal{N}(\omega)} \psi(t)\right)+\int_{0}^{\xi} \frac{(\xi-\eta)^{\omega-1}}{\Gamma(\omega)} \mathscr{H}(\eta, \phi(\eta)) d \eta \\
& +\frac{\omega}{\mathcal{N}(\omega) \Gamma(\omega)} \int_{0}^{t}(t-\eta)^{\omega-1} \mathscr{G}(\eta, \phi(\eta)) d \eta \\
& +\frac{\omega}{\mathcal{N}(\omega) \Gamma(\omega)} \int_{0}^{t}(t-\eta)^{\omega-1} \psi(\eta) d \eta .
\end{aligned}
$$

Moreover, from (9), we have

$$
\begin{aligned}
\mid \phi(t) & -\left[\mathscr{F}(t, \phi(t))+\frac{(1-\omega)}{\mathcal{N}(\omega)} \mathscr{G}(t, \phi(t))+\int_{0}^{\xi} \frac{(\xi-\eta)^{\omega-1}}{\Gamma(\omega)} \mathscr{H}(\eta, \phi(\eta)) d \eta\right. \\
& \left.+\frac{\omega}{\mathcal{N}(\omega) \Gamma(\omega)} \int_{0}^{t}(t-\eta)^{\omega-1} \mathscr{G}(\eta, \phi(\eta)) d \eta\right] \mid \\
\leq & \left(\frac{(1-\omega)}{\mathcal{N}(\omega)}+\frac{\omega \xi^{\omega}}{\mathcal{N}(\omega) \Gamma(\omega+1)}\right) \varepsilon .
\end{aligned}
$$

Proof 4 Thanks to Lemma 2, we obtain solution (9). Further, thanks to Remark 1, result (10) is obvious.

Theorem 4 Under Lemma 3, the solution of considered H-FDE (1) is H-U and generalized $H-U$ stable if $\Upsilon<1$ exists.

Proof 5 If $\phi \in \mathscr{Z}$ is any solution of problem (1) and $\bar{\phi} \in \mathscr{Z}$ is at most one result of (1), then consider

$$
\begin{aligned}
& \|\phi-\bar{\phi}\|_{\mathscr{Z}}=\max _{t \in \mathscr{J}}|\phi(t)-\bar{\phi}(t)| \\
& =\max _{t \in \mathscr{J}} \mid \phi(t)-\left[\mathscr{F}(t, \bar{\phi}(t))+\frac{(1-\omega)}{\mathcal{N}(\omega)} \mathscr{G}(t, \bar{\phi}(t))\right. \\
& +\int_{0}^{\xi} \frac{(\xi-\eta)^{\omega-1}}{\Gamma(\omega)} \mathscr{H}(\eta, \bar{\phi}(\eta)) d \eta \\
& \left.+\frac{\omega}{\mathcal{N}(\omega) \Gamma(\omega)} \int_{0}^{t}(t-\eta)^{\omega-1} \mathscr{G}(\eta, \bar{\phi}(\eta)) d \eta\right] \mid \\
& =\max _{t \in \mathscr{J}} \mid \phi(t)-\left[\mathscr{F}(t, \phi(t))+\frac{(1-\omega)}{\mathcal{N}(\omega)} \mathscr{G}(t, \phi(t))\right. \\
& +\int_{0}^{\xi} \frac{(\xi-\eta)^{\omega-1}}{\Gamma(\omega)} \mathscr{H}(\eta, \phi(\eta)) d \eta \\
& \left.+\frac{\omega}{\mathcal{N}(\omega) \Gamma(\omega)} \int_{0}^{t}(t-\eta)^{\omega-1} \mathscr{G}(\eta, \phi(\eta)) d \eta\right] \\
& +\left(\mathscr{F}(t, \phi(t))+\frac{(1-\omega)}{\mathcal{N}(\omega)} \mathscr{G}(t, \phi(t))+\int_{0}^{\xi} \frac{(\xi-\eta)^{\omega-1}}{\Gamma(\omega)} \mathscr{H}(\eta, \phi(\eta)) d \eta\right. \\
& \left.+\frac{\omega}{\mathcal{N}(\omega) \Gamma(\omega)} \int_{0}^{t}(t-\eta)^{\omega-1} \mathscr{G}(\eta, \phi(\eta)) d \eta\right)
\end{aligned}
$$




$$
\begin{aligned}
& -\left(\mathscr{F}(t, \bar{\phi}(t))+\frac{(1-\omega)}{\mathcal{N}(\omega)} \mathscr{G}(t, \bar{\phi}(t))+\int_{0}^{\xi} \frac{(\xi-\eta)^{\omega-1}}{\Gamma(\omega)} \mathscr{H}(\eta, \bar{\phi}(\eta)) d \eta\right. \\
& \left.+\frac{\omega}{\mathcal{N}(\omega) \Gamma(\omega)} \int_{0}^{t}(t-\eta)^{\omega-1} \mathscr{G}(\eta, \bar{\phi}(\eta)) d \eta\right) \mid
\end{aligned}
$$

Hence we get on using $\Omega_{1}=\frac{(1-\omega)}{\mathcal{N}(\omega)}+\frac{\omega \xi^{\omega}}{\mathcal{N}(\omega) \Gamma(\omega+1)}$

$$
\begin{aligned}
\|\phi-\bar{\phi}\|_{\mathscr{Z} \leq} \leq & \max _{t \in \mathscr{J}} \mid \phi(t)-\left[\mathscr{F}(t, \phi(t))+\frac{(1-\omega)}{\mathcal{N}(\omega)} \mathscr{G}(t, \phi(t))\right. \\
& +\int_{0}^{\xi} \frac{(\xi-\eta)^{\omega-1}}{\Gamma(\omega)} \mathscr{H}(\eta, \phi(\eta)) d \eta \\
& \left.+\frac{\omega}{\mathcal{N}(\omega) \Gamma(\omega)} \int_{0}^{t}(t-\eta)^{\omega-1} \mathscr{G}(\eta, \phi(\eta)) d \eta\right] \mid \\
& +\max _{t \in \mathscr{J}}|\mathscr{F}(t, \phi(t))-\mathscr{F}(t, \bar{\phi}(t))|+\frac{(1-\omega)}{\mathcal{N}(\omega)} \max _{t \in \mathscr{J}}|\mathscr{G}(t, \phi(t))-\mathscr{G}(t, \bar{\phi}(t))| \\
& +\max _{t \in \mathscr{J}} \int_{0}^{\xi} \frac{(\xi-\eta)^{\omega-1}}{\Gamma(\omega)}|\mathscr{H}(\eta, \phi(\eta))-\mathscr{H}(\eta, \bar{\phi}(\eta))| d \eta .
\end{aligned}
$$

Using hypothesis $\left(A_{1}\right)$, after some simplification in (12), we obtain by using Lemma 3

$$
\begin{aligned}
\|\phi-\bar{\phi}\|_{\mathscr{Z}} \leq & \Omega_{1} \varepsilon \\
& +\left[L_{\mathscr{F}}+\left(\frac{(1-\omega)}{\mathcal{N}(\omega)}+\frac{\omega \xi^{\omega}}{\mathcal{N}(\omega) \Gamma(\omega+1)}\right) L_{\mathscr{G}}\right. \\
& \left.+\frac{\xi^{\omega} L_{\mathscr{H}}}{\Gamma(\omega+1)}\right]\|\phi-\bar{\phi}\|_{\mathscr{Z}} .
\end{aligned}
$$

Hence, we get from (13)

$$
\|\phi-\bar{\phi}\|_{\mathscr{Z}} \leq \frac{\Omega_{1}}{1-\Upsilon} \varepsilon .
$$

Thus, the solution is $\mathrm{H}-\mathrm{U}$ stable. Further, for generalized $\mathrm{H}-\mathrm{U}$ stability, we have

$$
\mathscr{W}_{\mathscr{F}}=\frac{\Omega_{1}}{1-\Upsilon},
$$

and there exists a nondecreasing function $\varphi \in C((0,1),(0, \infty))$. Then from (14) we get

$$
\|\phi-\bar{\phi}\|_{\mathscr{Z}} \leq \mathscr{W}_{\mathscr{F}} \varphi(\varepsilon) \quad \text { with } \varphi(0)=0 .
$$

So the required result for generalized $\mathrm{H}-\mathrm{U}$ stability is received.

\section{Example of our analysis}

In this part, we justify our results via examples as follows.

Example 1 Consider the given H-FDE

$$
\left\{\begin{array}{l}
{ }_{0}^{A B C} \mathrm{D}_{t}^{\frac{1}{2}}\left[\phi(t)-\frac{\cos |\phi(t)|}{35}\right]=\frac{t^{3}+\sin |\phi(t)|}{45}, \quad t \in[0,1], \\
\phi(0)=\int_{0}^{1} \frac{(1-\eta)^{\omega-1}}{\Gamma(\omega)} \frac{1}{25} \exp (-\phi(\eta)) d \eta,
\end{array}\right.
$$


where

$$
\mathscr{F}(t, \phi(t))=\frac{\cos |\phi(t)|}{35}, \quad \mathscr{G}(t, \phi(t))=\frac{t^{3}+\sin |\phi(t)|}{45}
$$

and $\mathscr{H}(t, \phi(t))=\frac{1}{25} \exp (-\phi(t))$. As $\xi=1$ and $\omega=\frac{1}{2}$, let $\phi, \bar{\phi} \in \mathscr{Z}$, one has

$$
\begin{aligned}
|\mathscr{F}(t, \phi(t))-\mathscr{F}(t, \bar{\phi}(t))| & =\left|\frac{\cos |\phi(t)|}{35}-\frac{\cos |\bar{\phi}(t)|}{35}\right| \\
& \leq \frac{1}{35}|\phi(t)-\bar{\phi}(t)|, \\
|\mathscr{G}(t, \phi(t))-\mathscr{G}(t, \bar{\phi}(t))| & =\left|\frac{t^{3}+\sin |\phi(t)|}{45}-\frac{t^{3}+\sin |\bar{\phi}(t)|}{45}\right| \\
& \leq \frac{1}{45}|\phi(t)-\bar{\phi}(t)|
\end{aligned}
$$

and

$$
\begin{aligned}
|\mathscr{H}(t, \phi(t))-\mathscr{H}(t, \bar{\phi}(t))| & =\left|\frac{1}{25} \exp (-\phi(t))-\frac{1}{25} \exp (-\bar{\phi}(t))\right| \\
& \leq \frac{1}{25}|\phi(t)-\bar{\phi}(t)| .
\end{aligned}
$$

Thus we have $L_{f}=\frac{1}{35}, L_{g}=\frac{1}{45}$, and $L_{h}=\frac{1}{25}$. Now we check the conditions of the theorems and obtain

$$
\Upsilon=L_{\mathscr{F}}+\frac{1-\omega}{\mathcal{N}(\omega)} L_{\mathscr{G}}+\frac{L_{\mathscr{H}} \xi^{\omega}}{\Gamma(\omega+1)}\left(1+\frac{\omega}{\mathcal{N}(\omega)}\right)=\frac{409}{6300}<1 .
$$

Thus, the condition of Theorem 2 is satisfied. Therefore, problem (15) has a unique solution. Next

$$
L_{\mathscr{F}}+\frac{1-\omega}{\mathcal{N}(\omega)} L_{\mathscr{G}}=\frac{25}{630}<1 .
$$

Thus, the condition of Theorem 3 is also satisfied, hence the given problem (15) has at least one solution. Also for $\mathrm{H}-\mathrm{U}$ stability

$$
L_{\mathscr{F}}+\left(\frac{1-\omega}{\mathcal{N}(\omega)}+\frac{\omega \xi^{\omega}}{\mathcal{N}(\omega) \Gamma(\omega+1)}\right) L_{\mathscr{G}}+\frac{\xi^{\omega} L_{\mathscr{H}}}{\Gamma(\omega+1)}=\frac{377}{3150} \approx 0.11968<1 .
$$

Thus problem (15) is $\mathrm{H}-\mathrm{U}$ and generalized $\mathrm{H}-\mathrm{U}$ stable.

\section{Conclusion}

This work has successfully developed existence and stability results for the integral BVP of H-FDEs under nonsingular kernel type derivative. The concerned $\mathrm{BC}$ is nonlinear of integral type. Such problems have many applications in mathematical modeling of thermal and hydrodynamical problems. Based on fixed point approach, we have established some adequate results for at least one solution. Also some interesting results for $\mathrm{H}-\mathrm{U}$ type stability have been derived. The aforesaid derived results have been justified by providing 
a suitable problem. In the future, the aforesaid analysis can be extended to a nonlinear coupled system with integral BCs involving a Mittag-Leffler type derivative.

\author{
Acknowledgements \\ This research was funded by the Deanship of Scientific Research at Princess Nourah bint Abdulrahman University through \\ the Fast-track Research Funding Program.
}

\title{
Funding
}

This research was funded by Princess Nourah bint Abdulrahman University.

\section{Abbreviations}

Not applicable.

Availability of data and materials

Not applicable.

\section{Competing interests}

The authors declare that they have no competing interests.

\section{Authors' contributions}

All authors made equal contributions, read and supported the last original copy. All authors read and approved the final manuscript.

\section{Author details}

'Department of Mathematics, University of Malakand, Dir(L), Khyber Pakhtunkhwa, Pakistan. ${ }^{2}$ Department of Mathematical Sciences, Faculty of Science, Princess Nourah bint Abdulrahman University, P.O. Box 84428, Riyadh 11671, Saudi Arabia. ${ }^{3}$ Department of Mathematics, Cankaya University, Ankara, Turkey. ${ }^{4}$ Department of Medical Research, China Medical University Hospital, China Medical University, Taichung, Taiwan.

\section{Publisher's Note}

Springer Nature remains neutral with regard to jurisdictional claims in published maps and institutional affiliations.

Received: 28 February 2021 Accepted: 16 September 2021 Published online: 02 October 2021

\section{References}

1. Kilbas, A.A., Srivastava, H.M., Trujillo, J.J.: Theory and Applications of Fractional Differential Equations. Elsevier, Amsterdam (2006)

2. Podlubny, I.: Fractional Differential Equations: An Introduction to Fractional Derivatives, Fractional Differential Equations, to Methods of Their Solution and Some of Their Applications. Academic Press, New York (1998)

3. Abbas, S., Benchohra, M., N'Guérékata, G.M.: Topics in Fractional Differential Equations. Springer, New York (2012)

4. Rahimy, M.: Applications of fractional differential equations. Appl. Math. Sci. 4(50), 2453-2461 (2010)

5. Hilfer, R.: Applications of Fractional Calculus in Physics. World Scientific, Singapor (2000)

6. Dalir, M., Bashour, M.: Applications of fractional calculus. Appl. Math. Sci. 4(21), 1021-1032 (2010)

7. Lakshmikantham, V., Vatsala, A.S.: Basic theory of fractional differential equations. Nonlinear Anal., Theory Methods Appl. 69(8), 2677-2682 (2008)

8. Shah, K., Ali, A., Bushnaq, S.: Hyers-Ulam stability analysis to implicit Cauchy problem of fractional differential equations with impulsive conditions. Math. Methods Appl. Sci. 41(17), 8329-8343 (2018)

9. Ahmad, B., Luca, R.: Existence of solutions for a system of fractional differential equations with coupled nonlocal boundary conditions. Fract. Calc. Appl. Anal. 21(2), 423-441 (2018)

10. Dhage, B.C., Lakshmikantham, V.: Quadratic perturbations of periodic boundary value problems of second order ordinary differential equations. Differ. Equ. Appl. 2(4), 465-486 (2010)

11. Lakshmikantham, V., Vasundhara, D.J.: Theory of fractional differential equations in Banach space. Eur. J. Pure Appl. Math. 1(45), 38-45 (2008)

12. Hilal, K., Kajouni, A.: Boundary value problems for hybrid differential equations with fractional order. Adv. Differ. Equ. 2015, $183(2015)$

13. Hosseinnia, S.H., et al.: Experimental application of hybrid fractional-order adaptive cruise control at low speed. IEEE Trans. Control Syst. Technol. 22(6), 2329-2336 (2014)

14. Nouri, K., Baleanu, D., Torkzadeh, L.: Study on application of hybrid functions to fractional differential equations. Iran. J. Sci. Technol. Trans. A, Sci. 42(3), 1343-1350 (2018)

15. Houas, M.: Existence of solutions for a coupled system of Caputo-Hadamard type fractional differential equations with Hadamard fractional integral conditions. Adv. Theory Nonlinear Anal. Appl. 5(3), 316-329 (2021)

16. Nghia, B.: Existence of a mild solution to fractional differential equations with $\psi$-Caputo derivative and its $\psi$-Hölder continuity. Adv. Theory Nonlinear Anal. Appl. 5(3), 337-350 (2021)

17. Baitichea, Z., Derbazia, C., Benchohra, M.: $\psi$-Caputo fractional differential equations with multi-point boundary conditions by topological degree theory. Res. Nonlinear Anal. 3(4), 167-178 (2020)

18. Jarad, F., Abdeljawad, T.: A modified Laplace transform for certain generalized fractional operators. Res. Nonlinear Anal. 1(2), 88-98 (2018)

19. Tuan, N.H., Baleanu, D., Thach, T.N.: Final value problem for nonlinear time fractional reaction-diffusion equation with discrete data. J. Comput. Appl. Math. 376, 112-883 (2020) 
20. Hunga, N.N., Binhb, H.D., et al.: Stochastic sub-diffusion equation with conformable derivative driven by standard Brownian motion. Adv. Theory Nonlinear Anal. Appl. 5, 287-299 (2021)

21. Tuan, N.H., Huynh, L.N., Baleanu, D., Can, N.H.: On a terminal value problem for a generalization of the fractional diffusion equation with hyper-Bessel operator. Math. Methods Appl. Sci. 43(34), 2858-2882 (2020)

22. Caputo, M., Fabrizio, M.: A new definition of fractional derivative without singular kernel. Prog. Fract. Differ. Appl. 1(2), $1-13(2015)$

23. Atangana, A., Baleanu, D.: New fractional derivatives with nonlocal and non-singular kernel: theory and application to heat transfer model. Therm. Sci. 20, 763-769 (2016)

24. Algahtani, O.: Comparing the Atangana-Baleanu and Caputo-Fabrizio derivative with fractional order Allen Cahn model. Chaos Solitons Fractals 89(23), 552-559 (2016)

25. Abdeljawad, T:: A Lyapunov type inequality for fractional operators with nonsingular Mittag-Leffler kernel. J. Inequal. Appl. 2017, 130 (2017)

26. Tarasov, V.E.: Caputo-Fabrizio operator in terms of integer derivatives: memory or distributed lag. Comput. Appl. Math. 38(3), 113 (2019)

27. Shah, K., et al.: Semi-analytical study of Pine Wilt Disease model with convex rate under Caputo-Fabrizio fractional order derivative. Chaos Solitons Fractals 135, 109-754 (2020)

28. Wang, G., Pei, K., Chen, Y.: Stability analysis of nonlinear Hadamard fractional differential system. J. Franklin Inst. 356 6538-6546 (2019)

29. Abdeljawad, T:. Fractional operators with generalized Mittag-Leffler kernels and their iterated differintegrals. Chaos 29, 023102 (2019). https://doi.org/10.1063/1.5085726

30. Yavuz, M., Sulaiman, T.A., Yusuf, A., Abdeljawad, T.: The Schrodinger-KdV equation of fractional order with Mittag-Leffler nonsingular kernel. Alex. Eng. J. 60(2), 2715-2724 (2021)

31. Panda, S.K., Abdeljawad, T., Ravichandran, C.: Novel fixed point approach to Atangana-Baleanu fractional and $L^{p}$-Fredholm integral equations. Alex. Eng. J. 59(4), 1959-1970 (2020)

32. Miller, K., Ross, B.: Introduction to the Fractional Calculus and Fractional Differential Equations. Wiley, New York (1993)

33. Podlubny, l.: Fractional Differential Equations. Academic Press, San Diego (1999)

34. Tisdell, C.C.: Basic existence and a priori bound results for solutions to systems of boundary value problems for fractional differential equations. Electron. J. Differ. Equ. 2016, 84 (2016)

35. Ahmad, B., Nieto, J.J.: Existence of solutions for anti-periodic boundary value problems involving fractional differential equations via Leray-Schauder degree theory. Topol. Methods Nonlinear Anal. 35(2), 295-304 (2010)

36. Infante, G.: Eigenvalues and positive solutions of ODEs involving integral boundary conditions. Discrete Contin. Dyn. Syst. 2005, 436-442 (2005)

37. Ionkin, N.I: Solution of a boundary value problem in heat conduction theory with nonlocal boundary conditions. Differ. Equ. 13, 294-304 (1977)

38. Wang, J., Zhang, Y.: Ulam-Hyers-Mittag-Leffler stability of fractional-order delay differential equations. Fract. Syst. Optim. 63(8), 1181-1190 (2014)

39. Liu, K., Wang, J., O'Regan, D.: Ulam-Hyers-Mittag-Leffler stability for $\psi$-Hilfer fractional-order delay differentia equations. Adv. Differ. Equ. 2019, 50 (2019)

40. Kumama, P., Ali, A., Shah, K., Khan, R.A.: Existence results and Hyers-Ulam stability to a class of nonlinear arbitrary order differential equations. J. Nonlinear Sci. 10(6), 2986-2997 (2017)

41. Burton, T.A.: Krasnoselskii $N$-tupled fixed point theorem with applications to fractional nonlinear dynamical system. Adv. Math. Phys. 10(6), 2986-2997 (2019)

42. Jarad, F., Abdeljawad, T., Hammouch, Z:: On a class of ordinary differential equations in the frame of Atangana-Baleanu fractional derivative. Chaos Solitons Fractals 117, 16-20 (2018)

43. Ravichandran, C., Logeswari, K., Jarad, F.: New results on existence in the framework of Atangana-Baleanu derivative for fractional integro-differential equations. Chaos Solitons Fractals 125, 194-200 (2019)

44. Abdeljawad, T., Baleanu, D.: Discrete fractional differences with nonsingular discrete Mittag-Leffler kernels. Adv. Differ. Equ. 2016, 232 (2016). https://doi.org/10.1186/s13662-016-0949-5

45. Burton, T.A.: A fixed-point theorem of Krasnoselskii. Appl. Math. Lett. 11(1), 85-88 (1998)

\section{Submit your manuscript to a SpringerOpen ${ }^{\circ}$ journal and benefit from:}

- Convenient online submission

- Rigorous peer review

- Open access: articles freely available online

- High visibility within the field

- Retaining the copyright to your article

Submit your next manuscript at $\boldsymbol{~ s p r i n g e r o p e n . c o m ~}$ 